\title{
Clinical factors associated with susceptibility to infection stone formation: A Retrospective Single- Center Analysis
}

\section{Daming Wang}

Second Affiliated Hospital of Anhui Medical University

Demao Ding

second affiliated hospital of anhui medical university

Hongliang Sun

second affiliated hospital of anhui medical university

Lei Chen

second affiliated hospital of anhui medical university

\section{Zhiqiang Zhang}

Second Affiliated Hospital of Anhui Medical University

\section{Zhiqi Liu}

second affiliated hospital of anhui medical university

\section{Qinglin Ye}

Second Affiliated Hospital of Anhui Medical University

\section{Dazhao Zhang}

second affiliated hospital of anhui medical university

Dexin Yu ( $\nabla$ yudx_urology@126.com )

Dongdong Xie

second affiliated hospital of anhui medical university

\section{Research article}

Keywords: Infection stone; Clinical Susceptibility Factors; Struvite calculus; Apatite carbonate

Posted Date: March 5th, 2020

DOI: https://doi.org/10.21203/rs.3.rs-16116/v1

License: (c) (i) This work is licensed under a Creative Commons Attribution 4.0 International License.

Read Full License 


\section{Abstract}

Background: To investigate the clinical factors associated with susceptibility to infection stone formation and to analyze the variations in the clinical characteristics of stones with different compositions.

Methods: A total of 1213 patients were included in the study. The patients were grouped based on stone composition; 581 cases of infection stones (ammonium magnesium phosphate calculi or apatite carbonate content more than $50 \%$ ), 418 stones were composed of pure calcium oxalate, and 214 uric acid stone. The associations of infection stones with age, sex, body mass index, habits, working environment, recurrent stones, diabetes, hypertension, urinary tract infection, urine culture, urine $\mathrm{pH}$, and triglycerides were determined using logistic regression analysis. The variations in the clinical characteristics of the 3 groups were analyzed using analysis of variance or the chi-square test.

Results: The occurrence of infection stones was significantly associated with the patient's age (odds ratio [OR]: 1.031, 95\% confidence interval [Cl]: 1.020-1.041, P<0.001), high urine pH (OR: 1.974, 95\% Cl: $1.357-$ $2.872, \mathrm{P}<0.001)$, urinary tract infection (OR: $1.438,95 \% \mathrm{Cl}: 1.072-1.928, \mathrm{P}=0.015)$, positive urine cultures (OR: 1.541, 95\%Cl: 1.143-2.078, $\mathrm{P}=0.005$ ), and recurrent stones (OR: 1.346, 95\% Cl: 1.045-1.734, $\mathrm{P}=0.022)$, other clinical factors have no correlation. The clinical characteristics of the three groups were significantly different. The occurrence of infection stones is significantly associated with age, urinary tract infection, positive urine cultures, urine $\mathrm{pH}$, and stone recurrence.

Conclusions: Compared with previous studies, this study found that infection stones tend to occur in younger patients and stones resulting from non-urease-producing bacteria have significantly increased.

\section{Background}

Infection stone, otherwise known as struvite calculus, refers to a stone resulting from infection by ureaseproducing microorganisms. Infection stones are primarily composed of magnesium ammonium phosphate and carbon apatite, and account for approximately $15 \%$ of all cases of urinary stones[1]. Infection stones grow rapidly and have a high recurrence rate. If left untreated, they are prone to result in the development of chronic pyelonephritis, and even renal failure and urinary sepsis[2]. Various surgical approaches may be utilized to the treatment of infection stones. The common postoperative complication is infection. In severe cases, patients may even experience septic shock in response to the infection. This study retrospectively analyzed the clinical factors influencing the susceptibility to the formation of infection stones, with the aim of providing advice on the treatment and prevention of infection stones, as well as preventing serious postoperative complications and stone recurrence.

\section{Materials And Methods}

Study population: Patients with upper urinary tract stones that were removed through percutaneous nephrolithotomy or ureteroscopy at our hospital between June 2015 and April 2019 were included. All patients come from the local area and have a long-term follow-up in our hospital. A total of 1213 patients 
were enrolled in the study. The compositions of stones in all patients were analyzed using an automated infrared spectrometer (second generation). 581 cases of infection stones were screened out according to the composition of calculi (ammonium magnesium phosphate calculi or apatite carbonate content more than $50 \%$ ), 418 cases of calcium oxalate stones (calcium oxalate monohydrate, calcium oxalate dihydrate), and 214 cases of uric acid stones. Without family history of genetic diseases and neurological damage. The renal tubular acidosis was eliminated by preoperative examination of blood electrolyte, blood gas analysis, urine $\mathrm{pH}$, bicarbonate, titratable acid and ammonia. The hyperparathyroidism was eliminated by the examination of blood calcium, parathyroid hormone and parathyroid ultrasound. Elimination of cystine calculus and ceftriaxone calculus.

Study methods: The clinical data of patients were collected and retrospectively analyzed. The collected baseline characteristics included general data: gender, age, body mass index; habits: smoking (more than 5 cigarettes a day, smoking age $>1$ year), drinking (at least twice a month, each liquor $>100 \mathrm{ml}$ or beer $>$ $1000 \mathrm{ml}$, drinking history is more than 1 year), working environment (indoor or outdoor); medical history including duration of the stones (the time from stone discovery to stone treatment), recurrent stones (the patient had at least one stone recurrence, and the original stone was not completely removed before the recurrence was excluded), menopause, diabetes, hypertension; Test indicators: urine routine, urine bacterial culture (urine bacterial culture positive is bacteria were detected by collecting clean-voided midstream urine or puncture urine through renal pelvis for 48 hours), serum creatinine, serum uric acid, triglyceride. The associations between infection stones and each clinical factor were analyzed, and the clinical characteristics of infection stones, calcium oxalate stones, and uric acid stones were examined.

Statistical analysis: All data were analyzed and processed using the SPSS19.0 statistical software. Kolmogorov Smirnov method is used to test whether the measurement data conform to the normal distribution. Levene test is used to test the homogeneity of variance. All experimental data were showed as. Binary logistic regression analysis was used to evaluate the associations between each clinical variable and the occurrence of infection stones. Differences with a value of $\mathrm{P}<0.05$ were considered statistically significant. Intergroup comparisons were analyzed using the analysis of variance (ANOVA). Comparisons of categorical data among groups were analyzed using the chi-square test.

\section{Results}

- Analysis of baseline characteristics.

A total of 1213 patients with upper urinary tract stones were included in the study, comprising 758 men and 455 women. The age ranged from (20-84) years, with mean age was $51.47 \pm 12.86$ years, and the mean body mass index (BMI) was $24.02 \pm 3.12 \mathrm{~kg} / \mathrm{m}^{2}$. There were 112 patients with diabetes, 296 patients with hypertension, 924 patients with urinary tract infection, and 293 patients with positive urine cultures.

- Age distribution and bacterial spectrum of infectious stones. 
The age distribution of infectious stones mostly concentrated in 40-59 years old. (Figure1). In the infection stone group, there were 169 cases with positive urine cultures, and the most common bacteria were Escherichia coli (68 cases), Proteus mirabilis (29 cases), and Streptococcus agalactiae (11 cases). (Figure 2).

- Analysis of clinical factors and infection stones.

The stone composition analysis in this study revealed a total of 581 patients with infection stones. Logistic regression analysis was performed to examine the associations of infection stones with age, sex, $\mathrm{BMI}$, recurrent stones, duration of stones, diabetes, hypertension, triglyceride, urinary tract infection, urine culture, urine $\mathrm{pH}$, and living habits (smoking, drinking), working environment (indoor or outdoor). The results showed that the occurrence of infection stones was significantly associated with the patient's age (odds ratio [OR]: 1.031, 95\% confidence interval [Cl]: 1.020-1.041, P<0.001), urine pH (OR: 1.974, 95\% Cl: 1.357-2.872, $\mathrm{P}<0.001$ ), urinary tract infection (OR: 1.438, 95\% Cl: 1.072-1.928, $\mathrm{P}=0.015$ ), positive urine cultures (OR: 1.541, 95\%Cl: 1.143-2.078, P = 0.005), and recurrent stones (OR: 1.346, 95\% Cl: 1.045$1.734, P=0.022)$. There were no significant associations with sex, BMI, duration of stones, diabetes, hypertension, triglycerides, living habits and working environment. (Table 1).

- Comparisons of clinical characteristics in patients with the 3 types of stone compositions.

The patients were divided into 3 groups based on stone composition. The results of the chi-square test showed that the 3 groups had significant differences with respect to sex, first-time/recurrent stones, diabetes, hypertension, urinary tract infection, and urine bacterial culture positivity. ANOVA revealed significant differences in age, BMI, serum uric acid, creatinine, triglycerides and urine $\mathrm{pH}$ among the 3 groups. The age of infective stone group was the youngest and that of uric acid stone group was the oldest. The number of males in the three groups was higher than that of females, but the proportion of females (42.2\%) in the infection stone group was higher than that in the other two groups. BMI of uric acid stone group was significantly higher than that of the other two groups. The proportion of recurrent stones $(61.1 \%)$ was the highest in the infection stone group. The number of patients with diabetes and hypertension in the uric acid stone group was higher than that in the other two groups. The number of cases of urinary tract infection in the infection stone group was significantly higher than that in the other two groups. Urinary culture was positive in 169 cases (29.1\%) of infection stone group, which was significantly higher than the other two groups. The average urinary $\mathrm{pH}$ value in the infectious stone group was significantly higher than that in the other two groups. The average levels of serum uric acid, creatinine and triglyceride were the highest in the uric acid stone group (Table 2).

\section{Discussion And Conclusion}

The formation of infection stones mainly occurs owing to the continuous production of ammonia and carbon dioxide during urea decomposition by the urease enzyme produced by urea-splitting microorganisms in urine. The produced ammonia is removed in water and becomes ammonium, leading to an alkaline urine. Under this alkaline environment, ammonium, together with magnesium, phosphate, 
and water, can form magnesium ammonium phosphate hexahydrate crystals. Meanwhile, the carbonate produced by carbon dioxide and calcium and phosphate ions in urine form carbonate apatite crystals, which lead to the formation of infection stones[3]. Conventional research and analyses have shown that the common urease-producing bacteria include Proteus, Klebsiella, Pseudomonas, and Staphylococcus. In particular, Proteus mirabilis is the most common urease-producing bacteria. Meanwhile, $55 \%$ of Staphylococcus and only $1.4 \%$ of Escherichia coli produce urease[3, 4]. Previous studies found that bacterial cultures could be used to predict stone compositions, as stones with $>80 \%$ struvite content always contained urea-splitting bacteria, whereas unconventional urease-producing bacteria were mostly isolated from stones with $<20 \%$ struvite content[5]. Recent studies on the morphology of bacteria in infection stones have shown that the prevalence of stones resulting from non-urease-producing bacteria have been increasing[6]. The study of Paonessa showed that the urine and stone cultures of patients treated with percutaneous nephrolithotomy and reported that non-urease-producing bacteria were isolated in $23 \%$ of urine samples from patients with struvite stones[7]. Parkhomenko et al. also examined the bacterial profile of struvite stones using a stringent definition ( $>50 \%$ Struvite composition) and found that the traditional urease-producing bacteria were detected in only half of the positive stone cultures, whereas the isolation rates of Escherichia coli and Enterococcus were $18 \%$ and $12 \%$, respectively[8]. The increased incidence of Escherichia coli in patients with infection stones is caused by multiple infections, as Escherichia coli is a relatively common pathogen owing to its short replication time. In addition, studies have indicated that plasmid-mediated transfer of the urease gene may also be a possible mechanism of this observation[5]. In the present study, the detection rate of Escherichia coli was also the highest in the infection stone group. The occurrence of infection stones was significantly associated with positive urine cultures and urinary tract infection. The incidence of urinary tract infection and positive bacterial cultures was significantly higher in the infection stone group than in the other 2 groups, indicating that patients with infection stones had both prior and ongoing urinary tract infection.

In all 3 groups of this study, the number of male patients was higher than that of female patients. Men were more susceptible to stone diseases than women; however, the proportion of women was highest in the infection stone group (42.2\%) than in the calcium oxalate stone group (33.0\%) and the uric acid stone group (33.6\%). Daudon et al. have shown that the stone composition in male patients is predominantly calcium oxalate and uric acid, whereas female patients mainly harbor infection stones[9]. With improvements in medical quality, the current incidence of infection stones has significantly decreased. Marickar and Vijay have demonstrated that although the incidence of stone diseases has significantly increased in recent years, the incidence of infection stones in female patients has significantly decreased[10]. Similarly, the reduction in the incidence of staghorn calculi in Europe supports the notion that infection stones constitute the majority of large stones[2]. Previous studies have shown that the incidence of infection stones is higher in older patients; however, a recent study suggested that infection stones tend to occur more frequently in younger patients, whereas the incidence of uric acid stones significantly increases with age[9]. The present study found that age was significantly associated with the occurrence of infection stones, with patients in the infection stone group being significantly younger than those in the other 2 groups. Krambeck et al. have also indicated that infection stones are more 
common in younger patients and uric acid stones are more common in older patients. As uric acid stones are related to increased age, decreased urine $\mathrm{pH}$, kidney aging, and environmental changes[11]. The present study found that the uric acid stone group had significantly older age, higher serum creatinine, and higher serum uric acid than the other 2 groups, whereas urine $\mathrm{pH}$ was significantly lower in the uric acid stone group than in the other groups. These results fully support the above viewpoints.

This study also found that the proportion of recurrent stones in the infection stone group $(61.1 \%)$ was significantly greater than that in the calcium oxalate stone group (54.3\%) and the uric acid stone group $(49.1 \%)$. Stone recurrence was significantly associated with infection stones. Infection stones are prone to recur. Infection stones grow extremely fast and can be formed in only 4-6 weeks, with a recurrence rate of up to $50 \%[3]$. Furthermore, some studies have shown that calcium oxalate stones have a strong tendency to be converted into calcium phosphate stones, and kidney stone phosphorylation is a key factor in stone recurrence[12,13]. Zeng et al. also demonstrated that the stone compositions changed over time in patients with recurrent stones[14]. A survey showed that $32.9 \%$ of patients had alterations in stone compositions, with no sex differences. A change of the major component between calcium oxalate and infection stones was found in $15.7 \%$ of calcium oxalate stones and $51.7 \%$ of infection stones, and infection stones could change into uric acid or calcium oxalate stones.

Although diabetes may easily cause or aggravate urinary tract infection that leads to urinary stones and infections, the disease itself is not significantly associated with the occurrence of infection stones. Studies have shown that the proportion of uric acid stones is significantly higher in diabetic patients than in non-diabetic patients[15-17]. Patients with diabetes have been demonstrated to have lower urine $\mathrm{pH}$, as the glucose in their urine competitively inhibits uric acid reabsorption. The excretion and saturation of urate and oxalate are higher in patients with diabetes than in the those without. In addition, insulin resistance promotes changes in hydrolase in the 24-h urine, leading to more acidic urine and lower urinary citrate levels, which are important risk factors for stone formation. Patients with diabetes commonly harbor uric acid and calcium oxalate stones. The present study also confirmed these observations. There was no association between diabetes and infection stones, and the proportion of patients with diabetes in the uric acid stone group (20.9\%) was significantly higher than that in the calcium oxalate stone group (7.9\%) and the infection stone group (6.2\%). In addition, this study found no associations between the occurrence of infection stones and hypertension, $\mathrm{BMI}$, and triglycerides. There were no differences in BMI and triglycerides among the 3 groups studied. The number of patients with hypertension in the uric acid group (38.8\%) was significantly higher than that in the calcium oxalate stone group $(23.7 \%)$ and the infection stone group (19.6\%). This could be related to the low urinary citrate and high uric acid excretion in patients with primary hypertension, which favor the formation of uric acid stones[18, 19].

Infectious Urinary Stones can lead to end stage renal disease either from obstructive nephropathy with staghorn calculi or from recurrent pyelonephritis, the urease enzyme and resultant high urine pH caused by microorganisms in struvite stone formers may also contribute to kidney injury, large staghorn calculi may also cause papillary necrosis ${ }^{19}$. However, even if the obstruction is relieved and the stone is 
removed, the renal damage caused by infectious stones is still persistent. Early detection and treatment of renal injury is very important.

The deficiency of this study is a single center retrospective study, with a small sample size and limited in the region. There is limited information on bacteriology because of the differences between the results of calculus and urine bacterial culture[20]. We hope to strengthen multicenter cooperation and increase sample size and regional differences in future research.

Collectively, the occurrence of infection stones is significantly associated with age, urinary tract infection, positive urine cultures, urine $\mathrm{pH}$, and stone recurrence. Compared with previous studies, this study found that infection stones tend to occur in younger patients and stones resulting from non-urease-producing bacteria have significantly increased. In addition, infection stones are not associated with sex, diabetes, hypertension, BMI, or hyperlipidemia. This study revealed the factors associated with the susceptibility to infection stone formation and the variations in clinical characteristics of infection stones compared with other types of stones. These results can provide better guidance on the screening, treatment, and prevention of postoperative complications.

\section{Declarations}

Abbreviations:

OR: odds ratio

Cl: confidence interval

BMI: body mass index

Ethics approval and consent to participate: This article does not contain any studies with human participants or animals performed by any of the authors.

Consent for publication: Written informed consent was obtained from the patient for publication of this case report and any accompanying images. A copy of the written consent is available for review by the Editor of this journal.

Availability of data and material: The datasets used during this study available from the corresponding author on reasonable request.

Competing interests: The authors declare that they have no conflict of interests.

Funding: This article does not contain any Foundation.

Authors' contributions: All authors read and approved the manuscript to publish. All authors help to draft or revise the manuscript. 
DM Wang: Manuscript writing, Data collection and management, Data analysis

DM Ding: Manuscript editing, Protocol, Data analysis, Project development

HL Sun: Data collection and management, Manuscript editing

DD Xie: Data management, Manuscript editing

L Chen: Data collection and management

ZQ Zhang: Manuscript editing, Data analysis

ZQ Liu: Data collection

QL Ye: Data collection

DZ Zhang: Data collection and management

DX Yu: Project development, Manuscript editing, Protocol

Acknowledgements: no applicable

Author's Contribution

DM Wang: Manuscript writing, Data collection and management, Data analysis

DM Ding: Manuscript editing, Protocol, Data analysis, Project development

HL Sun: Data collection and management, Manuscript editing

L Chen: Data collection and management

ZQ Zhang: Manuscript editing, Data analysis

ZQ Liu: Data collection

QL Ye: Data collection

DZ Zhang: Data collection and management

DX Yu: Project development, Manuscript editing, Protocol

DD Xie: Data management, Manuscript editing

\section{References}


1.Thomas B, Tolley D: Concurrent urinary tract infection and stone disease: pathogenesis, diagnosis and management. Nat Clin Pract Urol 2008, 5(12):668-675.

2.Preminger GM, Assimos DG, Lingeman JE, Nakada SY, Pearle MS, Wolf JJ: Chapter 1: AUA guideline on management of staghorn calculi: diagnosis and treatment recommendations. J Urol 2005, 173(6):19912000 .

3.Bichler KH, Eipper E, Naber K, Braun V, Zimmermann R, Lahme S: Urinary infection stones. Int J Antimicrob Agents 2002, 19(6):488-498.

4.Gault MH, Longerich LL, Crane G, Cooper R, Dow D, Best L, Stockall E, Brown W: Bacteriology of urinary tract stones. J Urol 1995, 153(4):1164-1170.

5.Flannigan R, Choy WH, Chew B, Lange D: Renal struvite stones-pathogenesis, microbiology, and management strategies. NAT REV UROL 2014, 11(6):333-341.

6.Nevo A, Shahait M, Shah A, Jackman S, Averch T: Defining a clinically significant struvite stone: a nonrandomized retrospective study. INT UROL NEPHROL 2019, 51(4):585-591.

7.Paonessa JE, Gnessin E, Bhojani N, Williams JJ, Lingeman JE: Preoperative Bladder Urine Culture as a Predictor of Intraoperative Stone Culture Results: Clinical Implications and Relationship to Stone Composition. J Urol 2016, 196(3):769-774.

8.Parkhomenko E, De Fazio A, Tran T, Thai J, Blum K, Gupta M: A Multi-Institutional Study of Struvite Stones: Patterns of Infection and Colonization. J ENDOUROL 2017, 31(5):533-537.

9.Daudon M, Dore JC, Jungers P, Lacour B: Changes in stone composition according to age and gender of patients: a multivariate epidemiological approach. Urol Res 2004, 32(3):241-247.

10.Marickar YM, Vijay A: Female stone disease: the changing trend. Urol Res 2009, 37(6):337-340.

11.Krambeck AE, Lieske JC, Li X, Bergstralh EJ, Melton LR, Rule AD: Effect of age on the clinical presentation of incident symptomatic urolithiasis in the general population. J Urol 2013, 189(1):158-164.

12.Aihara K, Byer KJ, Khan SR: Calcium phosphate-induced renal epithelial injury and stone formation: involvement of reactive oxygen species. KIDNEY INT 2003, 64(4):1283-1291.

13.Mandel N, Mandel I, Fryjoff K, Rejniak T, Mandel G: Conversion of calcium oxalate to calcium phosphate with recurrent stone episodes. J Urol 2003, 169(6):2026-2029.

14.Zeng G, Zhao Z, Wu W, Ou L, Liang Y, Yuan J: Interconversion of stone composition profiles from two recurrent stone episodes in stone formers. CLIN CHEM LAB MED 2014, 52(7):1019-1024.

15. Weinberg AE, Patel CJ, Chertow GM, Leppert JT: Diabetic severity and risk of kidney stone disease. EUR UROL 2014, 65(1):242-247. 
16.Zhu W, Mai Z, Qin J, Duan X, Liu Y, Zhao Z, Yuan J, Wan SP, Zeng G: Difference in 24-Hour Urine Composition between Diabetic and Non-Diabetic Adults without Nephrolithiasis. PLOS ONE 2016, 11(2):e150006.

17.Nerli R, Jali M, Guntaka AK, Patne P, Patil S, Hiremath MB: Type 2 diabetes mellitus and renal stones. Adv Biomed Res 2015, 4:180.

18. Hartman C, Friedlander JI, Moreira DM, Leavitt DA, Hoenig DM, Smith AD, Okeke Z: Does hypertension impact 24-hour urine parameters in patients with nephrolithiasis? UROLOGY 2015, 85(3):539-543.

19.Losito A, Nunzi EG, Covarelli C, Nunzi E, Ferrara G: Increased acid excretion in kidney stone formers with essential hypertension. Nephrol Dial Transplant 2009, 24(1):137-141.

20.Walton-Diaz A, Vinay JI, Barahona J, Daels P, González M, Hidalgo JP, Palma C, Díaz P, Domenech A, Valenzuela $\mathrm{R}$ et al: Concordance of renal stone culture: PMUC, RPUC, RSC and post-PCNL sepsis-a nonrandomized prospective observation cohort study. INT UROL NEPHROL 2017, 49(1):31-35.

\section{Tables}

Due to technical limitations, all tables are only available for download from the Supplementary Files section.

\section{Figures}




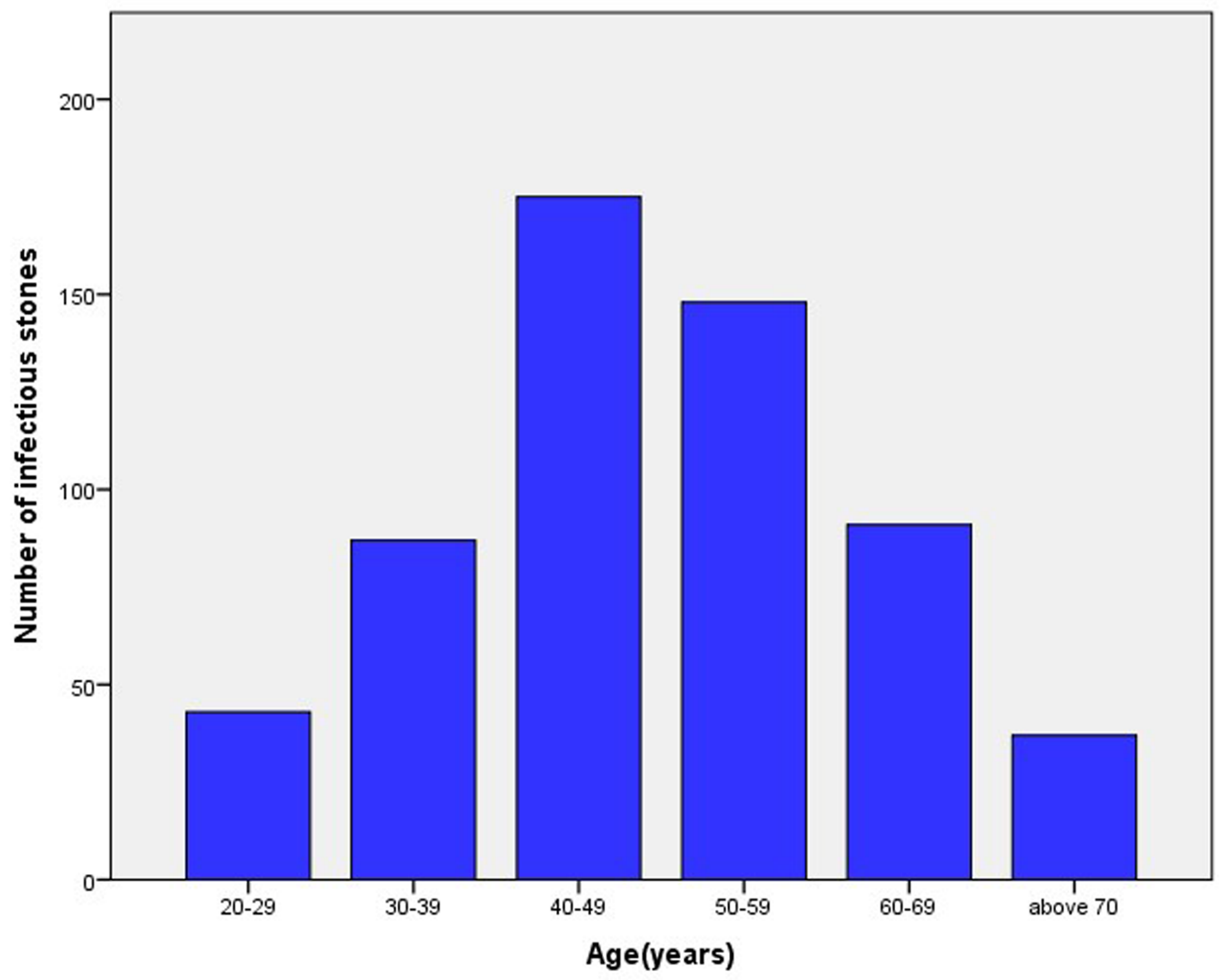

Figure 1

Age Distribution Characteristics of Infectious Stones. The age distribution of infectious stones mostly concentrated in 40-59 years old. 


\section{Number of cases}

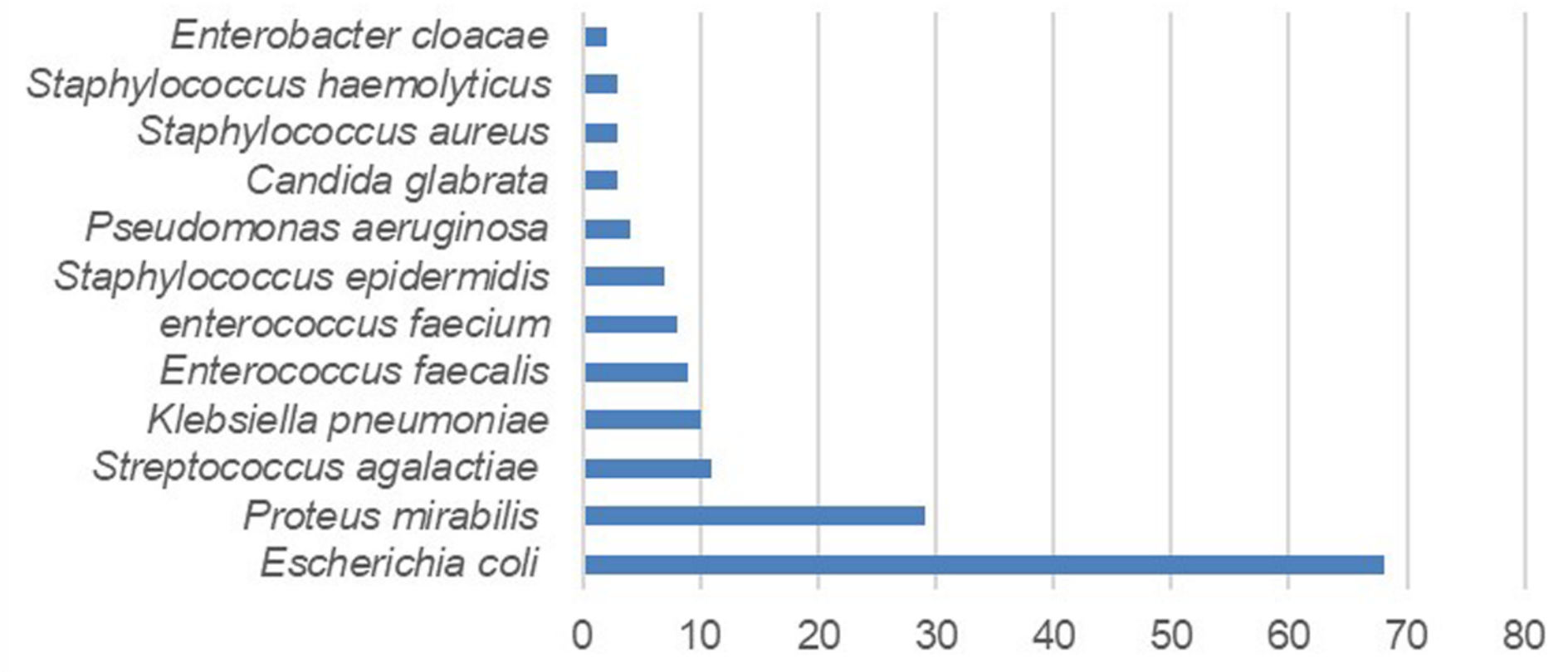

Figure 2

Bacterial spectrum of infectious calculi. In the infection stone group, there were 169 cases with positive urine cultures, and the most common bacteria were Escherichia coli (68 cases), Proteus mirabilis (29 cases), and Streptococcus agalactiae (11 cases).

\section{Supplementary Files}

This is a list of supplementary files associated with this preprint. Click to download.

- Table2.docx

- Table1.docx 